Supporting Material for

\title{
Dynamics Across a Free Surface Reflect Interplay Between Density and Cooperative Length: Application to Polystyrene
}

\author{
Ronald P. White and Jane E.G. Lipson* \\ Department of Chemistry, \\ Dartmouth College, Hanover, NH 03755
}

\section{A. LCL Equation of State Application to Bulk Polystyrene: Free Volume Analysis and Modeling of the Local Density Profile}

In order to apply the cooperative free volume (CFV) rate model $\tau\left(T, V_{\text {free }}\right)$ expression to the dynamics of polystyrene (PS), we first need to predict the free volume values of PS from its characteristic thermodynamic $(P V T)$ data. Free volume is defined ${ }^{1}$ as $V_{\text {free }}(T, P)=V(T, P)-V_{\mathrm{hc}}$, where $V$ is a system's overall volume and $V_{\text {hc }}$ is its limiting value at close packing. To obtain $V_{\text {hc }}$, we apply the locally correlated lattice (LCL) equation of state (EOS) ${ }^{1}$ which is given by

$$
\begin{gathered}
\frac{P}{k_{\mathrm{B}} T}=\left(\frac{1}{v}\right) \ln \left[\frac{V}{V-N_{\mathrm{m}} r v}\right]+\left(\frac{3}{v}\right) \ln \left[\frac{V-\left(N_{\mathrm{m}} v / 3\right)(r-1)}{V}\right] \\
-\left(\frac{3}{v}\right)\left(\frac{(2 r+1)^{2}}{\left(V / N_{\mathrm{m}} v\right)-(1 / 3)(r-1)}\right) \times\left(\frac{\exp \left[-\varepsilon / k_{\mathrm{B}} T\right]-1}{(1 / 3)(2 r+1) \exp \left[-\varepsilon / k_{\mathrm{B}} T\right]+\left(V / N_{\mathrm{m}} v\right)-r}\right)
\end{gathered}
$$

where $k_{\mathrm{B}}$ is the Boltzmann constant and $N_{\mathrm{m}}$ is the number of molecules. (In practice, we calculate the volume per molecule or per gram given the molecular weight.) The molecular parameters are as follows. $r$ is the number of segments (occupied lattice sites) per molecule, $v$ is the volume per lattice site, and $\varepsilon$ is the segment-segment nonbonded interaction energy. $r, v, \varepsilon$ are obtained by fitting to $P V T$ data and this leads to the characteristic volume at close-packing, which is given by $V_{\mathrm{hc}}=N_{\mathrm{m}} r v$, or equivalently in volume per gram, $V_{\mathrm{hc}}=r v / M_{\mathrm{w}}$ where $M_{\mathrm{w}}$ is the molecular weight in $\mathrm{g} / \mathrm{mol}$ and where $v$ is in volume per mol.

The LCL EOS was fit to PS PVT data $\left(M_{\mathrm{w}}=110,000 \mathrm{~g} / \mathrm{mol}\right)$ from Zoller and Walsh ${ }^{2}$ in a $T$ range of 384 to $463 \mathrm{~K}$ and $P$ range of 0 to $100 \mathrm{MPa}$, which is reasonably close to the relevant 
range of the PS dynamics data. A plot of the fit is shown in Figure S1. The result of the fit gives the characteristic parameters: $r / M_{\mathrm{w}}=0.11529 \mathrm{~mol} / \mathrm{g}, v=7.5621 \mathrm{~mL} / \mathrm{mol}, \varepsilon=-2136 \mathrm{~J} / \mathrm{mol}$. From the $r$ and $v$ values we thus obtain a close-packed hard-core volume of $V_{\mathrm{hc}}=r v / M_{\mathrm{w}}=0.8718 \mathrm{~mL} / \mathrm{g}$. Note when we apply this value of $V_{\mathrm{hc}}$ to calculate free volumes, $V_{\text {free }}(T, P)=V(T, P)-V_{\mathrm{hc}}$, at each $T, P$ point (e.g. at a chosen dynamics $T, P$ data point), we use the LCL EOS $V(T, P)$ value at that $T, P$. The LCL $V(T, P)$ is essentially equivalent to the true experimental volume when the $T, P$ is close to the $P V T$ fitting data range, so a distinction only arises when outside the fitting range. For the latter case we have found that it is important to maintain the match of the theoretical $V(T, P)$ and theoretical $V_{\text {hc }}$ for consistency. The LCL $V(T, P)$ may become inaccurate if the $(T, P)$ of interest lie well outside the fitted range, however, use of the LCL values for both $V(T, P)$ and $V_{\text {he }}$ leads to a cancellation/compensation of errors and allows us to apply the free volume analysis over a range that is much wider than the $P V T$ data fitting range.

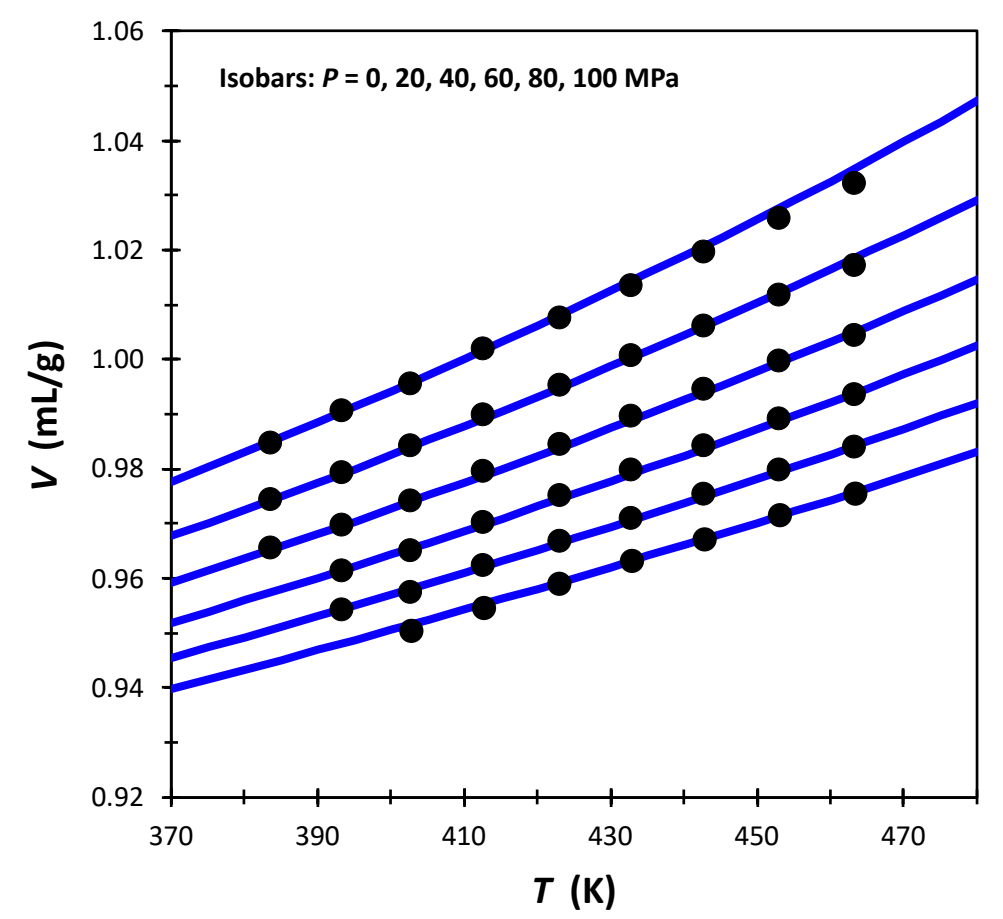

Figure S1. LCL equation of state fit to polystyrene pressure-volume-temperature data. LCL model shown as curves, experimental data as points. (Data from Zoller and Walsh, ${ }^{2}$ PS molecular weight, $M_{\mathrm{w}}=110,000$ g/mol.) 
Based on the characterization of the PS PVT behavior, the LCL EOS was also applied to calculate the local density profile for the PS free surface. This is done by combining the LCL EOS with the square gradient approximation. ${ }^{3-5}$ Here the Helmholtz free energy density at a given position, $z$, near the free surface is given by $a(\rho(z))=a_{0}(\rho(z))+\kappa(\nabla \rho(z))^{2}$. This is a sum of the homogenous contribution, $a_{0}(\rho(z))$, plus a single gradient term (with parameter $\kappa$ ) to account for the inhomogeneous contribution coming from the locally varying density. The homogeneous free energy density, $a_{0}(\rho(z))$, is supplied by the LCL EOS for the given $\rho$ value, and with a value of the parameter $\kappa$, the surface tension, $\sigma$, and local density profile, $\rho(z)$, can then be calculated. These calculations involve integrations carried out over the variable, $\rho$, and are described in detail in refs ${ }^{3-5}$. Here for PS, we fit the experimental surface tension to obtain what is effectively the value of $\kappa$, and this then leads to the corresponding density profile for that fitted $\kappa$. Figure S2 shows a plot of the model fit of PS experimental surface tension data, $\sigma$ as a function of $T$, where data are taken from $\mathrm{Wu}^{6}$. In Figure $\mathrm{S} 3$ we show the corresponding model results for the PS free surface local density profile, $\rho(z)$, at three different temperatures; the profiles are sharp (about $1 \mathrm{~nm}$ wide), broadening just slightly as $T$ increases.



Figure S2. LCL EOS with square gradient approximation, model fit of the gradient parameter to polystyrene surface tension data. Model curve shown as a function of temperature, experimental data shown as points. 


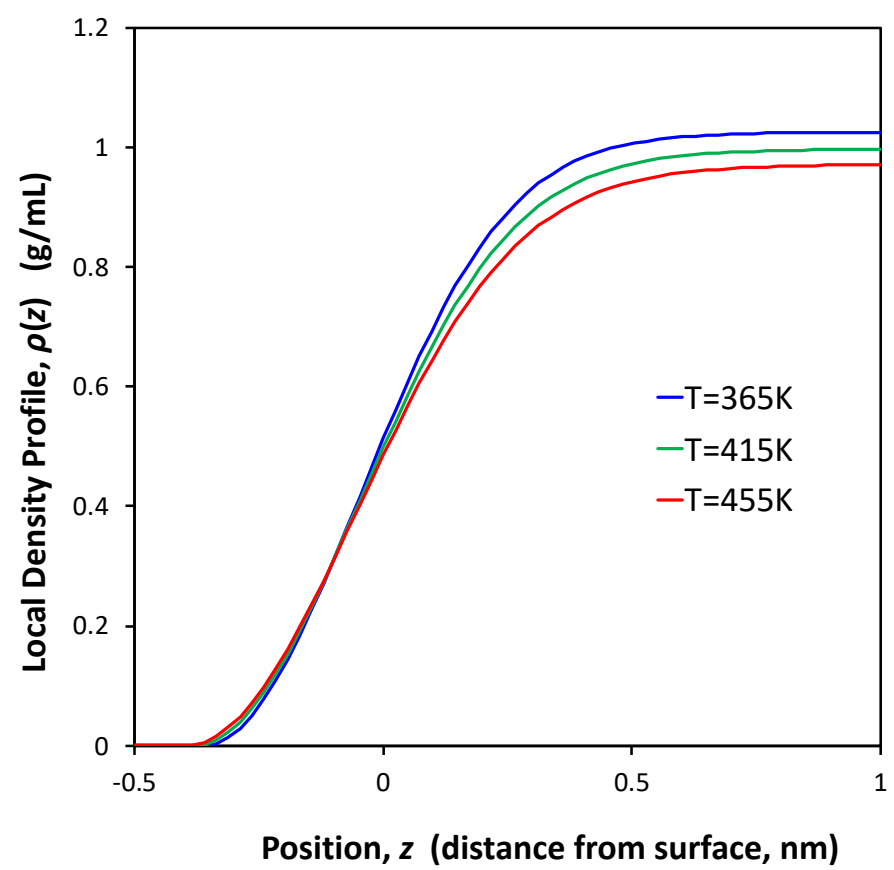

Figure S3. LCL EOS with square gradient approximation, local density profiles, $\rho(z)$, as a function of position, $z$, at three temperatures, $T=365,415$, and $455 \mathrm{~K}$.

\section{B. Modeling Bulk Polystyrene Dynamics Using the Cooperative Free Volume Rate Model}

The CFV model expression for segmental relaxation times is given by

$$
\ln \tau=\left(\frac{V_{\mathrm{hc}}}{V_{\text {free }}}\right)\left(\frac{T^{*}}{T}\right)^{b}+\ln \tau_{\text {ref }}
$$

The equation applies to dynamics under general pressure dependent conditions, where $V_{\text {free }}(T, P)=$ $V(T, P)-V_{\mathrm{hc}}$ at any given $T, P$, comes from the LCL EOS analysis of $P V T$ data. The three material specific parameters that are based on bulk system dynamics data are $b, T^{*}$, and $\tau_{\text {ref. }}$ While our main goal is to apply the CFV model to bulk and interfacial environments under just ambient pressure conditions, it is still very important to make sure that the individual effects from temperature and volume are properly represented ( $T$ and $V$ are both changing as $T$ varies at ambient $P$ ). The system's characteristic relative sensitivity to changes in $T$ is controled by the $b$ parameter, which is defined as $b=-\left(\partial \ln V_{\text {free }} / \partial \ln T\right)_{\tau}$. The most reliable way to ensure that a fitted $b$ value is 
representative of the system is to utilize pressure dependent data. (Note that Eq. S2 is not perfect, and therefore fitting all three parameters $b, T^{*}, \tau_{\text {ref, }}$, to just an ambient pressure dynamics data set could potentially "overfit" the $b$ value, so using some pressure dependent information prevents this possibility.)

Shown in Figure S4 are some available pressure-dependent dynamics data for polystyrene from Schwartz et al. ${ }^{7} P$-dependent dielectric spectroscopy measurements on PS are challenging, so the trends in the higher pressure data are not as smooth as might be observed in other systems.

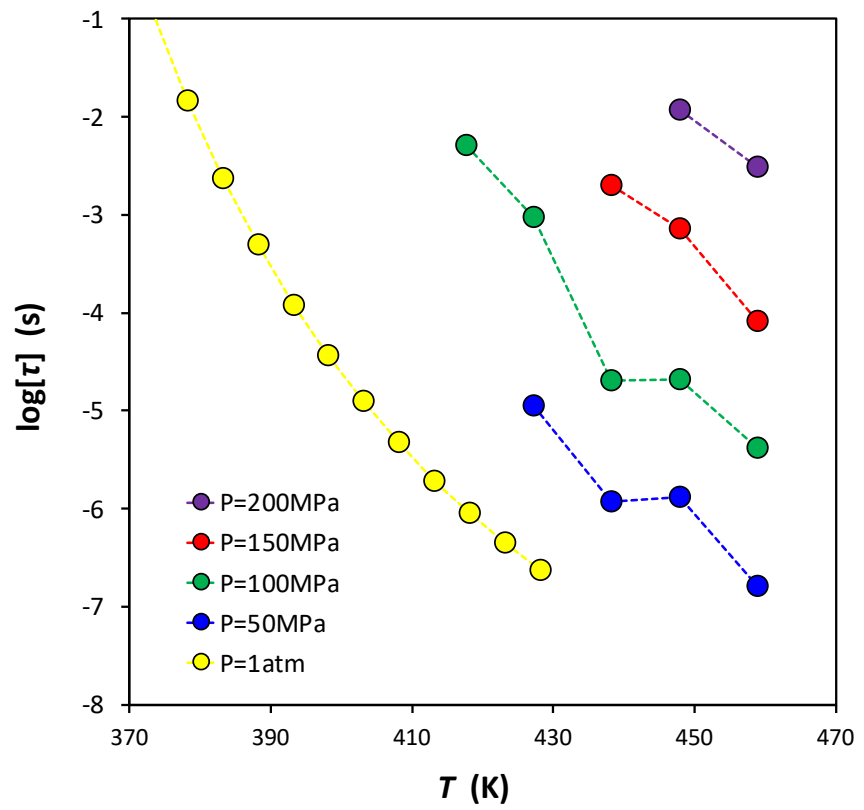

Figure S4. Polystyrene pressure dependent dynamics data ( $\alpha$-relaxation times via dielectric spectroscopy) taken from Schwartz et al. ${ }^{7} \log \tau$ as a function of $T$ on isobars at $P=1 \mathrm{~atm}$, and higher pressures of 50, 100, 150 , and $200 \mathrm{MPa}$.

Equation S2 shows that the CFV $b$ parameter for PS can be determined by collapsing the data in Figure S4 in a plot of $\log \tau$ vs. $1 /\left(V_{\text {free }} T^{b}\right)$. With the correct value for $b$ (e.g. found by simple trial and error), the plot is expected to form a single line, and the remaining parameters $T^{*}$ and $\tau_{\text {ref }}$ follow from the line's slope and intercept. This collapsed line, corresponding to a value of $b=$ 2.55 , is shown in the left panel of Figure S5. (An alternative equivalent route it is to do a least 
squares fit of eq S2 of all three parameters $\left(b, T^{*}, \tau_{\text {ref }}\right)$ simultaneously; this approach is actually the source of $b=2.55$ value demonstrated in the plot here.) The data collapse in Figure S5 is not as tight as for other systems we have treated (e.g. see ref ${ }^{8}$ ); this is expected however, given the appearance of the data in Figure S4. As a comparison, we also applied the density scaling approach $^{9-12}$ to the same PS data, where a plot of $\log \tau$ vs. $1 / T V^{\gamma}$ is expected to collapse into a single curve with the correct value of the material dependent parameter, $\gamma$. This plot is shown in the right panel of Figure S4; as expected the collapse is not very tight; the plot corresponds to a best $\gamma$ value of 3.75 .
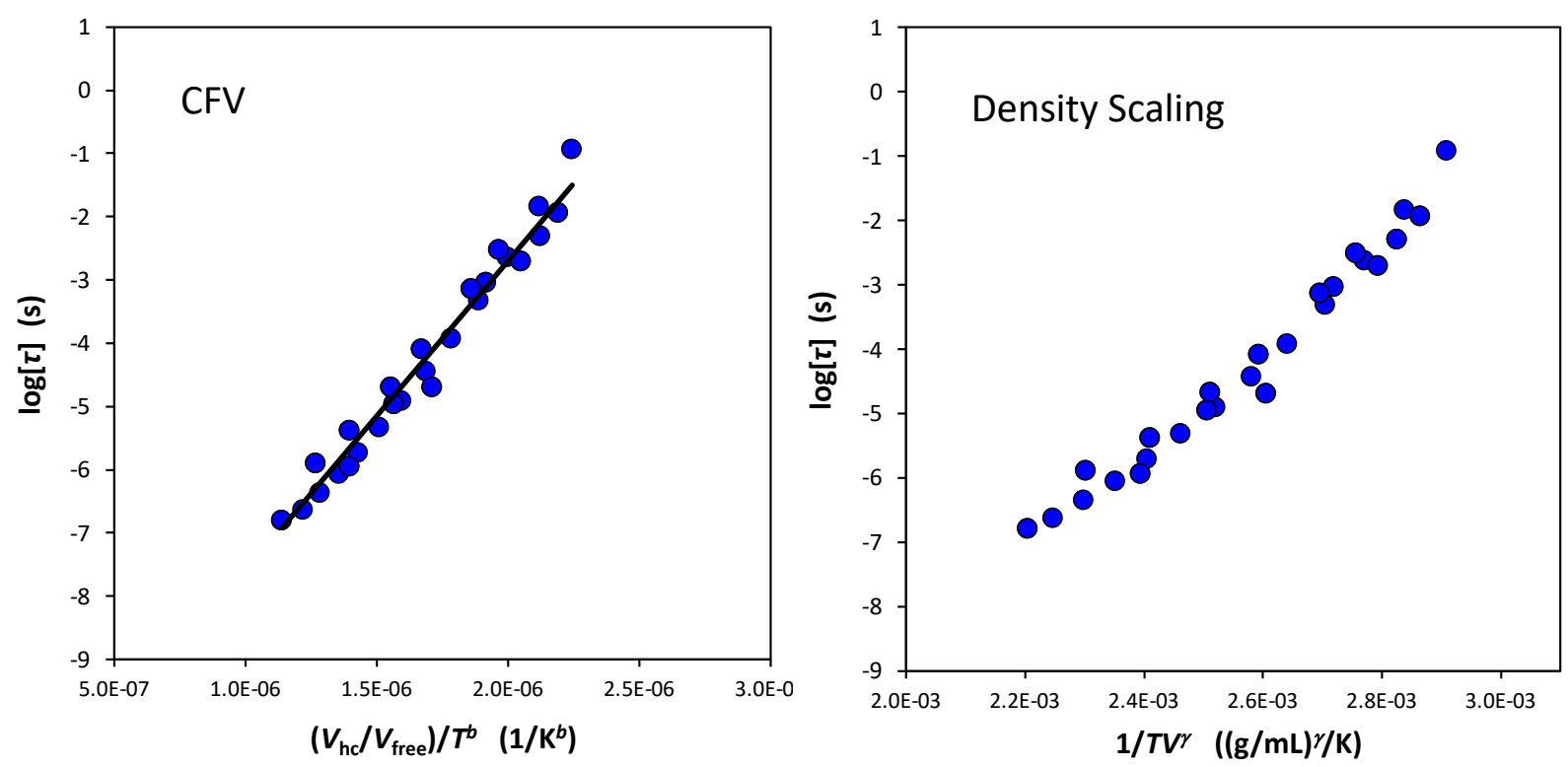

Figure S5. Model collapse of polystyrene pressure dependent dynamics data from Figure S4. CFV model results in the left panel show $\log \tau$ vs. $\left(V_{\text {hd }} / V_{\text {free }}\right) / T^{b}$, with $b=2.55$ (close to, but somewhat smaller than the $b$ value from $P V T$ data, 3.21). Density scaling results in the right panel show $\log \tau$ vs. $1 /\left(T V^{\prime}\right)$, with $\gamma=$ 3.75 (close to, but somewhat larger than the $\gamma$ value from PVT data, 3.47).

To increase the reliability of our determination of the $b$ parameter, we added additional information from the pressure dependence of the glass transition temperature $\left(T_{\mathrm{g}}\right)$ from $P V T$ data. The $T_{\mathrm{g}}(P)$ values represent an isochronal condition (all $T_{\mathrm{g}}$ points are expected to have the same relaxation time), and so, given that we know $V_{\text {free }}(T, P)$ from LCL EOS analysis, we can apply the $b=-\left(\partial \ln V_{\text {free }} / \partial \ln T\right)_{\tau}$ relationship to solve for $b$. Equivalently, this relationship can be expressed 
from eq $\mathrm{S} 2$ as $\left(T_{2} / T_{1}\right)^{b}=\left(V_{\text {free, } 1} / V_{\text {free, } 2}\right)$ for two states $(1$ and 2$)$ that have the same relaxation time. For more information, this procedure was described in detail in ref ${ }^{13}$, where we applied it to poly 4-chlorostyrene.
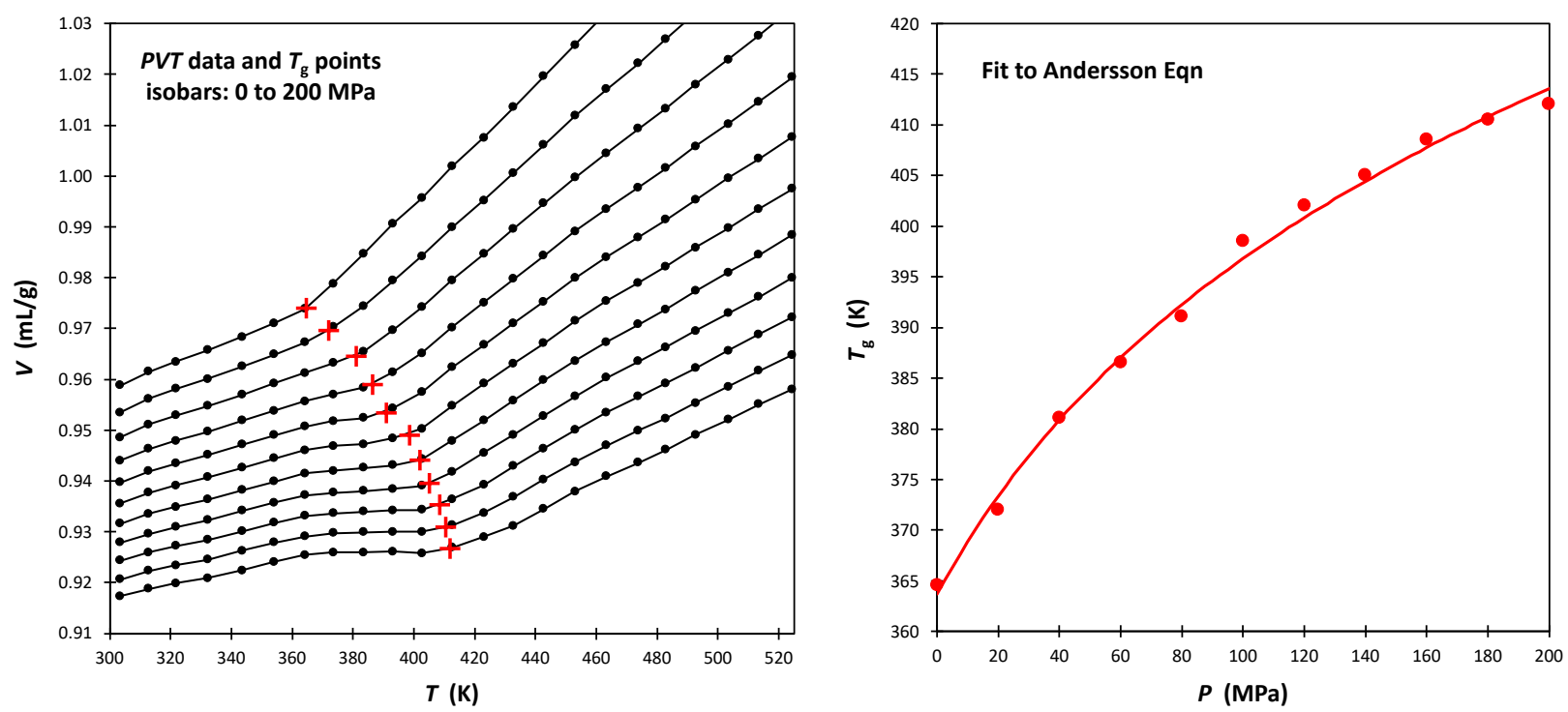

Figure S6. Analysis of the pressure dependence of $T_{\mathrm{g}}$ from $P V T$ data $^{2}\left(M_{\mathrm{w}}=110,000 \mathrm{~g} / \mathrm{mol}\right)$. The left panel shows PS PVT data, where, for each isobar, the red cross symbol marks the approximate location of $T_{\mathrm{g}}$, the point where there is deviation from the trend in liquid branch of $V(T)$ on decreasing $T$. The right panel shows a smooth fit of these $T_{\mathrm{g}}(P)$ points to the Andersson equation. ${ }^{14}$ These results lead to an estimate of $b=3.21$ for the CFV model (close to, but somewhat larger than the $b$ value from PVT data, 2.55), and $\gamma$ $=3.47$ for the density scaling model(close to, but somewhat smaller than the $\gamma$ value from $P V T$ data, 3.75).

In Figure S6, the left panel shows the PVT data covering melt and glassy regimes. The red crosses mark the approximate location of the $T_{\mathrm{g}}$ point, the point where, as $T$ decreases, there is deviation from the trend along the liquid branch of the isobaric $V(T)$ curve. These approximate $T_{\mathrm{g}}(P)$ points were then fit to a smooth form (the Andersson equation $\left.{ }^{14}\right)$. Taking the $T_{\mathrm{g}}(P)$ value from the smooth curve at $P=100 \mathrm{MPa}$ (chosen because it is roughly in the middle of the $P$ range), we then solve for $b$ from $\left(T_{2} / T_{1}\right)^{b}=\left(V_{\text {free, } 1} / V_{\text {free, } 2}\right)$, obtaining $b=3.21$. This is close to, but a little larger than the $b=2.55$ value determined from the $P$-dependent dielectric spectroscopy data. (Note that our studies of about a dozen different polymer and small molecule liquid systems show characterisitc $b$ values ranging from 1.6 to 6.4.) 


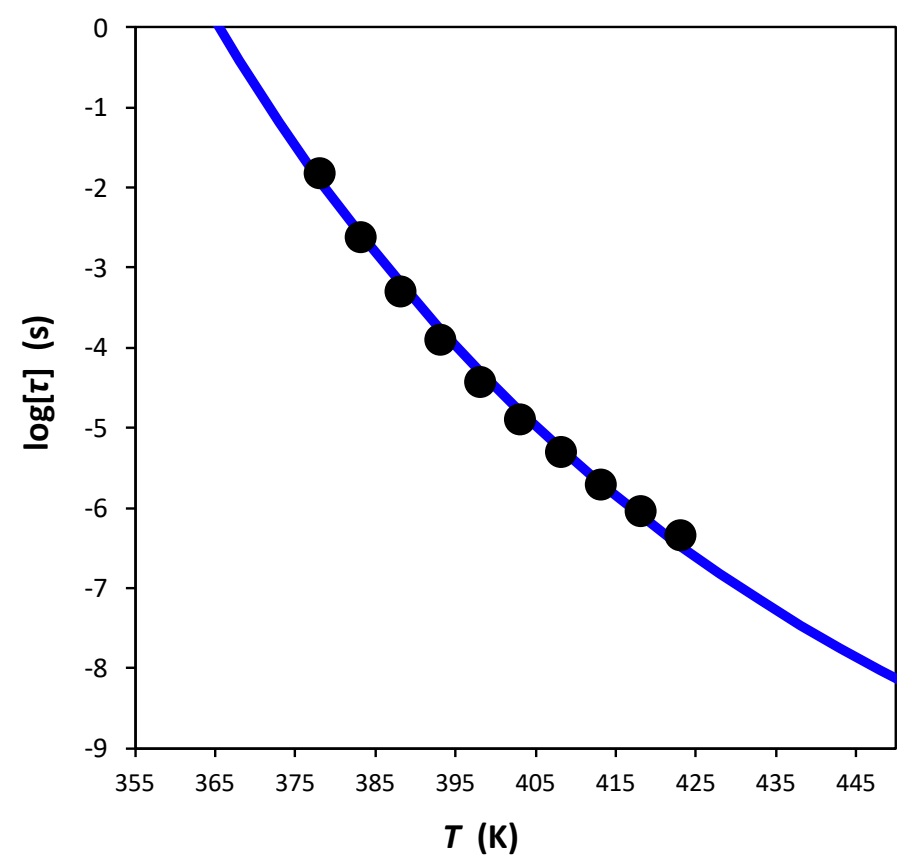

Figure S7. CFV model fit of the $T^{*}$ and $\tau_{\text {ref }}$ parameters to ambient pressure dynamics data, $\log \tau$ as a function of $T$, model shown as curve, and data as points The model curve corresponds to a complete parameters set of: $b=2.88, T^{*}=566 \mathrm{~K}, \log \tau_{\text {ref }}=-12.9$. The $b$ parameter of was fixed in this fit at the average best value (2.88) determined from dynamics data at higher pressure and $T_{\mathrm{g}}(P)$ from $P V T$ data.

From the two independent determinations of $b$ above (2.55 and 3.21), we then take the best value to be the average, $b=2.88$. Our interest in this work is to model PS films and bulk at ambient pressure, so it is at this pressure $(P=1 \mathrm{~atm})$ that we want the model to be the most accurate. Therefore we fix this $b=2.88$ value, and fit the remaining two CFV parameters to the smooth ambient pressure data from Schwartz, et al. ${ }^{7}$ and obtain $T^{*}=566 \mathrm{~K}$ and $\log \tau_{\text {ref }}=-12.9$. This fit is shown in Figure S7, which is quite sufficient for our purposes. As emphasized above, the balance between temperature and density contributions is important under ambient conditions because density changes with $T$ along the isobar, and further, at interfaces, the density changes with position. This is why we apply the CFV $P$-dependent dynamics model to represent the independent $T$ and $V$ contributions. 


\section{References}

(1) White, R. P.; Lipson, J. E. G. Polymer Free Volume and Its Connection to the Glass Transition. Macromolecules 2016, 49 (11), 3987-4007. https://doi.org/10.1021/acs.macromol.6b00215.

(2) Zoller, P.; Walsh, D. Standard Pressure-Volume-Temperature Data for Polymers; Technomic Pub Co.: Lancaster, PA, 1995.

(3) Sanchez, I. C. Physics of Polymer Surfaces and Interfaces; Butterworth: Greenwich, CT, 1992.

(4) Poser, C. I.; Sanchez, I. C. Surface-Tension Theory of Pure Liquids and Polymer Melts. J. Colloid Interface Sci. 1979, 69 (3), 539-548. https://doi.org/10.1016/00219797(79)90142-5.

(5) Dee, G.; Sauer, B. The Surface Tension of Polymer Liquids. Adv. Phys. 1998, 47 (2), 161205. https://doi.org/10.1080/000187398243546.

(6) Wu, S. Surface and Interfacial Tensions of Polymer Melts .2. Poly(Methyl Methacrylate), Poly(Normal-Butyl Methacrylate), and Polystyrene. J. Phys. Chem. 1970, 74 (3), 632-. https://doi.org/10.1021/j100698a026.

(7) Schwartz, G. A.; Colmenero, J.; Alegria, A. Dielectric Study of the Segmental Relaxation of Low and High Molecular Weight Polystyrenes under Hydrostatic Pressure. J. NonCryst. Solids 2007, 353 (47-51), 4298-4302. https://doi.org/10.1016/j.jnoncrysol.2007.02.070.

(8) White, R. P.; Lipson, J. E. G. The Cooperative Free Volume Rate Model for Segmental Dynamics: Application to Glass-Forming Liquids and Connections with the Density Scaling Approach. Eur. Phys. J. E 2019, 42 (8), 100. https://doi.org/10.1140/epje/i201911862-3.

(9) Casalini, R.; Mohanty, U.; Roland, C. M. Thermodynamic Interpretation of the Scaling of the Dynamics of Supercooled Liquids. J. Chem. Phys. 2006, 125 (1), 014505-014505. https://doi.org/10.1063/1.2206582.

(10) Casalini, R.; Roland, C. M. An Equation for the Description of Volume and Temperature Dependences of the Dynamics of Supercooled Liquids and Polymer Melts. J. Non-Cryst. Solids 2007, 353 (41-43), 3936-3939. https://doi.org/10.1016/j.jnoncrysol.2007.03.026.

(11) Grzybowski, A.; Paluch, M. Universality of Density Scaling. In The Scaling of Relaxation Processes; Kremer, F., Loidl, A., Eds.; Advances in Dielectrics; Springer International Publishing: Cham, 2018; pp 77-119. https://doi.org/10.1007/978-3-319-72706-6_4.

(12) Dyre, J. C. Hidden Scale Invariance in Condensed Matter. J. Phys. Chem. B 2014, 118 (34), 10007-10024. https://doi.org/10.1021/jp501852b.

(13) White, R. P.; Lipson, J. E. G. Connecting Pressure-Dependent Dynamics to Dynamics under Confinement: The Cooperative Free Volume Model Applied to Poly(4Chlorostyrene) Bulk and Thin Films. Macromolecules 2018, 51 (20), 7924-7941. https://doi.org/10.1021/acs.macromol.8b01392.

(14) Andersson, S.; Andersson, O. Relaxation Studies of Poly(Propylene Glycol) under High Pressure. Macromolecules 1998, 31 (9), 2999-3006. https://doi.org/10.1021/ma971282z. 